\title{
Solar Cell Imaging: A Gateway to Stem Disciplines
}

\section{Dr. Michael G Mauk P.E., Drexel University \\ Dr. Richard Chiou, Drexel University (Eng.) \\ Mr. M. Eric Carr, Drexel University}

Mr. Eric Carr is a full-time Laboratory Manager and part-time adjunct instructor with Drexel University's Department of Engineering Technology. Eric assists faculty members with the development and implementation of various Engineering Technology courses. A graduate of Old Dominion University's Computer Engineering Technology program and Drexel's College of Engineering, Eric enjoys finding innovative ways to use microcontrollers and other technologies to enhance Drexel's Engineering Technology course offerings. Eric is currently pursuing a Ph.D in Computer Engineering at Drexel, and is an author of several technical papers in the field of Engineering Technology Education. 


\title{
Imaging of Solar Cells:
}

\section{A Gateway to Teaching STEM Disciplines}

\author{
Abstract \\ In this project, we are using image processing (both visible, near infrared, and far \\ infrared) to study various aspects of solar cells including their materials, device \\ operation, defects, variability, and reliability. Laboratory projects using low-cost \\ fluorescent cameras, visible and near-IR cameras, and far-infrared thermal cameras are \\ used to characterize the grain structure, defects, surface roughness, reflectivity, \\ electroluminescence, photoluminescence, and photovoltaic operation of solar cell \\ materials (e.g., monocrystalline and multicrystalline silicon wafers), thin-film and nano \\ solar cells, commercial silicon solar cells, and photovoltaic modules. Students can \\ import captured images into MATLAB or other widely-available image processing \\ software for analysis and interpretation. Topical laboratory modules and projects are \\ being developed suitable for on-line delivery.
}

Overview, Purpose and Broad Aims: The purpose of this work is to develop, validate, and disseminate a series of laboratory/classroom educational modules that use image capture, image processing and image analysis of photovoltaic solar cells to teach concepts and methods in:

- materials science and semiconductor technology including image-based studies of electrical and optical properties and defects, and image analysis of features at several scales (submicron, micron, $\mathrm{mm}$ ), as well as packaging, area variability and yield.

- surface metrology (e.g., micro roughness, cleanliness and surface damage, etching uniformity, and characteristic geometries of microstructured surfaces)

- metallography (microstructure such as grain boundaries and texture, i.e., preferred grain orientations and size distributions and their impact on material performance)

- thin-film optics such as anti-reflection coatings

- many aspects of photovoltaics and other optoelectronic devices such as LEDs, sensors, flat panel displays, and other energy conversion devices such as photocathodes

- basic ideas of hyperspectral and remote imaging

- radiative, conductive, and convective heat transfer; and various heating and cooling methods (resistive heating and convective heating/cooling, thermoelectric (Peltier) effects, optical heating with lasers and flash lamps, ultrasonic heating)

- thermal and infrared physics, as particularly related to thermal imaging and IR optics 
- thermomechanical properties of materials, such as thermal expansion and thermalinduced stresses

- $\mathrm{CCD} / \mathrm{CMOS}$ cameras including cellphone and web cameras, thermal imaging cameras, and visible, UV and IR quantum and thermal detectors

- image capture, image processing, and image analysis such as edge sharpening and detection, skeletonization, segmentation, convolution and filtering, feature and pattern registration and recognition, mathematical morphology, and more advanced techniques.

- signal processing and signal extraction from noise, such as implemented with filtering, phase-sensitive detection and lock-in amplification techniques

- non-destructive testing including elucidation of subsurface features and defects

- robotics (pick and place, sorting, conveyer built inspection)

- machine vision for quality and process control

- reliability, as related to, for example, hot spots developed by printed circuit boards and solar modules operating in the field.

The skills and knowledge gained with these topics will reinforce a host of supporting and related STEM subjects, meeting the needs of a diverse undergraduate engineering audience. All of these topics can be profitably taught in a unified manner by analyzing solar cells or solar modules in various stages of fabrication using a CCD or CMOS camera, widely available image analysis software, and increasingly affordable highperformance thermal cameras. These educational modules may serve as the basis of courses in photovoltaics, image processing and machine vision, and non-destructive testing; or may supplement existing courses in quality control, robotics, semiconductor technology, and other allied and related fields. The work described here can be implemented at relatively modest costs, facilitating dissemination to a wide audience of students in STEM disciplines. Web-based laboratories where students remotely control an image capture session of fixtured solar cells and receive instrument data and images in real-time will also be developed, allowing thermal camera imaging analysis at any site with internet access. The methods taught here are of increasing importance in manufacturing, quality and reliability, process control, robotics and automation, homeland security, infrastructure inspection, maintenance, renewable energy, and biomedical fields including clinical diagnostics. Machine vision is being fully integrated into solar cell factories across the entire value chain: inspecting and controlling processes for ingots, wafers, thin-film deposition, texturing and etching, cleaning, screen printing, and coatings, in many cases at rates up to 2000 cells per hour. Other expanding uses of imaging include building inspection, weatherization, biometric recognition, fire fighting, inspection of welds, food processing, plastic injection molding, to name but a few applications. The use of image analysis for process control (temperature fields, and fluid velocity profiles) is assuming increasing importance in many industries [SCOTT and MCCANN 2005].

This work seeks to address several perceived needs in American education and industry. First, many aspects of image processing are often relegated to a specializations of electrical engineering, and similarly, machine vision is commonly focused on robotics and automation. We believe the image processing concepts taught in the proposed modules - especially as related to mapping areal variations of 
temperature (thermography), thermal effects, luminescence, reflection, transmission, optical scattering, and their intimate connections with microstructure, device design and operation, areal and process scale-up, performance, quality, and reliability--deserve a much wider audience among STEM students. Second, a review of the research and trade literature shows that the main efforts in these areas-particularly with regard to renewable energy, are in Germany, Japan, Korea, China, Australia, and the Netherlands, with comparatively less activity in the US. Of possible relevance, the recent German textbook Infrared Thermal Imaging: Fundamentals, Research and Applications (VolLMER and MölLMANN, 2010) describes how thermography in the broader context of visualization and pedagogy is used to teach many varied topics in science and technology in European schools and universities. Third, we believe the much greater utilization of imaging and analysis at the research, process and product development stages will speed up progress in renewable energy, nanotechnology, materials processing, medical diagnostics, and homeland security. Our broad aim is to help provide the next generation of students with the knowledge and skill sets to effectively use and contribute to this increasingly important area.

The proposed project will develop course materials and methods that will serve a diverse range of students, and which can be delivered in various formats for adaptation by a number of schools with a low investment in materials, equipment, and space. The solar cell as a case study provides a highly-accessible, safe, very topical, and scientifically interesting subject for imaging techniques, that will impress students with the richness and profound capabilities of imaging. The project will develop faculty expertise, through participation in Industry Workshops and classes (e.g., at the Infrared Training Center, Boston, MA). This work will enable many fulfilling projects for students in both science and engineering. Aspects of optical and thermal imaging for characterization of materials and processes, as a means for visualization and as a diagnostics and metrology tool will be emphasized.

Technical Basis. The solar cell is truly a microcosm of materials science and engineering, optics, and semiconductor physics and technology; and thus serves as an outstanding case study to elucidate the varied phenomena that can be studied by imaging techniques. We are hard pressed to think of any other type of specimen that offers such and so-many accessible 'windows' to the capabilities of imaging as a research and engineering tool-and with only a modest investment in equipment and lab space as will be described. Solar cells exhibit photoluminescence, electroluminescence, highly spectral- and angular-dependent reflectance, scattering and transmission, and pronounced thermal emission and absorption in the infrared — driven by electrical and optical injection and transport of charge carriers through diffusion, drift and thermoelectric (Peltier and Seebeck) effects, and a host of radiative and non-radiative carrier recombination processes, often mediated by various types of defects. Further, solar cells include features such screen printed metallization, various optical and passivating coatings and encapsulation, and surface texturing, all of which impose many interesting features to their images. Moreover, these effects are sensitive to operational conditions such as temperature, light intensity, electrical bias, and mechanical stress, as well as device design and materials characteristics related to impurities, oxidation, precipitates, grain size and texture, dislocations, and many types of defects. All of these effects vary over the area and surface(s) of the solar cell, and 
captured images of solar cells reveal, either directly or indirectly, the spatial distribution map of these effects and properties. Most crucial defect types have specific thermal and optical signatures which can be identified and characterized by imaging. Many new technologies, such as solar cells, solid-state lighting, superconductivity, medical imaging, as well as many proposed nanotechnologies, can falter in commercialization because they encounter serious difficulties when scaled up for large-area manufacture. Imaging provides tremendous capabilities and insights that cannot be achieved or realized with traditional "point sensors" such as temperature or voltage probes, or pressure and strain gauges, either in basic research and process engineering, as for example, to assess and assure areal uniformity of chemical, structural, optical, and electrical properties. Combined with statistical process control, embedded in manufacturing execution systems, such advanced monitoring, metrology and diagnostics afforded by imaging will allow better optimization, higher yields, and lower costs across many modern industries and business enterprises.

The solar cell proves an interesting for imaging because the effects and phenomena that can be imaged are indicative of properties (resistivity, reflectivity, carrier injection, recombination, and trapping, etc...) that crucially influence performance. Further, these image-capturable effects can be excited or modulated optically (with lasers, LED arrays or flash lamps), electrically (by voltage or current sources), thermally (with contact or probe heating), or ultrasonically using piezoelectric transducers. An important aspect of the proposed work is that one type of sample, namely a solar cell in various stages of fabrication, can be imaged by a very diverse set of techniques that corroborate, supplement, or complement each other. Students can then fully appreciate the essential connections between materials, optical, thermal, and electrical properties and means to probe these phenomena.

Many of the types of defects and anomalies investigated with solar cell imaging have similar or analogous counterparts in integrated circuits, printed circuit boards, thin-film displays, electrodes and catalysts, solid-state lighting, and large-area detectors. After completion of these modules, the student will be able to apply imaging to many diverse (and often unrelated) areas of technology. Further, although focused on solar cells and related optoelectronic devices and semiconductor materials as an exemplary application area for purposes of instruction and convenience, we believe these modules will nevertheless well prepare students for the kinds of image analysis needed for aircraft, automobiles, and buildings, as well as to biomedical diagnostics and industrial process control.

A captured image of a solar cell directly indicates the spatial variation of photoluminescence, electro-luminescence, reflectance, absorption, and localized heating through emission of infrared radiation, and indirectly the current, voltage, power, and temperature of the solar cell. By analyzing the raw image data (i.e., pixel intensities expressed as matrices), it is possible to map the localized temperature (and heating effects), surface roughness or microstructure (and light scattering), reflectivity (and coating uniformity), resistivity, minority carrier lifetime, defect densities, excess carrier concentration, carrier trapping effects, and so forth. Further analysis can discriminate between various material defects such as precipitates, dislocations, stacking faults, and grain boundaries. The solar cell thus provides an almost astounding variety of effects 
that can be imaged. Further, these are not esoteric effects of merely academic interest: all of these phenomena play an important role in determining the performance (i.e., energy conversion efficiency) and reliability of the solar cell. In order to assess the capabilities and current practices of imaging in R\&D and industry, particularly with respect to solar cells, the proposers reviewed over 450 research publications and review articles, plus company technical literature; and conferred with numerous machine vision and thermal camera providers regarding applications of image processing.

We survey a few of the solar cell imaging techniques that will form the basis of the educational modules developed here. Although space does not permit a detailed technical description of the methods and analysis of imaging, the foregoing should give the reviewers some appreciation of the

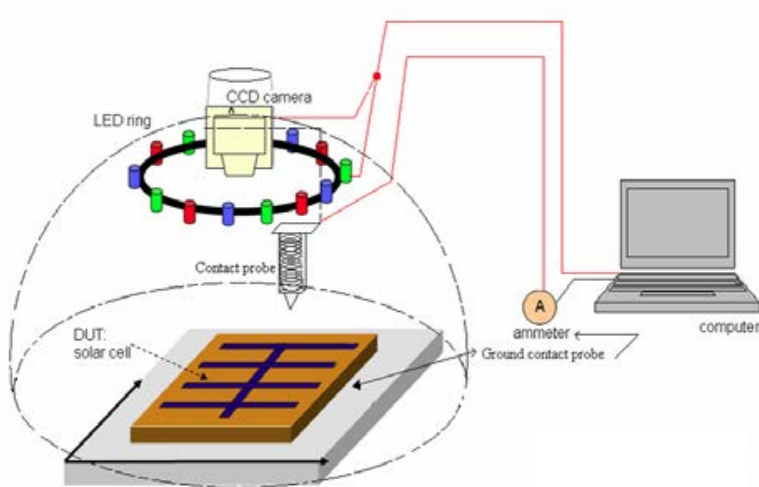

Figure 1: LED illumination for CCD imaging of solar cells. scope and levels of sophistication covered by the modules, and the knowledge students will gain by engaging in a subset of modules opted by their instructor or their own interests. Figure 1 shows a ring of LEDs and a stage that our undergraduates constructed to illuminate solar cells (or photovoltaic material samples) for image capture with a CCD camera. Some examples of images captured for analysis are shown in Figures $\mathbf{2}$ and $\mathbf{3}$ for multicrystalline silicon solar cells. This setup allows the user to control the wavelengths and angles of incident light on the solar cell. The grain boundaries are quite evident with multicolor illumination as each grain reflects a characteristic color due to its surface texture. In Figure 3, two images of a solar cell are taken under a white light source (white LEDs) at different incident angles, showing that features (in this case metallization lines), and defects in those features, are better contrasted for certain illumination conditions. It is interesting that the microstructure of silicon is so evident without any surface preparation, in contrast to the polishing and etching needed to bring out the grain structures of metal samples.

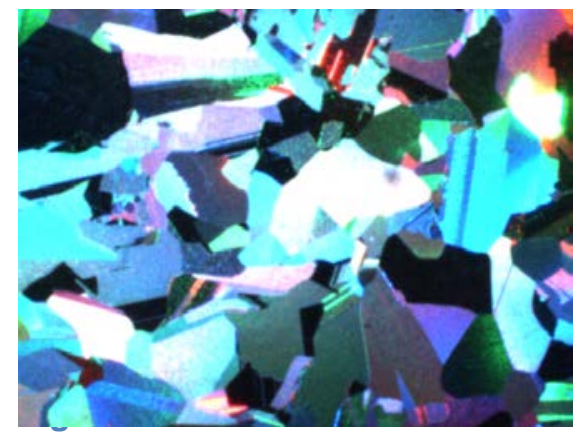

crystalline silicon wafer captured by CCD with multicolor LED ring illumination.
The image can be skeletonized along grain boundaries (Figure 4) by thresholding and creating a binary image; and the grain sizes can be ascertained by simple image processing methods such as in ImageJ by counting the number of black pixels. As a simple analysis, the cumulative grain size distribution can be plotted as in Figure 5. This is a good predictor of solar cell performance since the grain boundaries are electrically

active in silicon and represent a significant loss mechanism. The grain structure will also be evident in luminescence images discussed below, to the effect 
that a microstructural feature is shown closely related to optical phenomena. More elaborate microstructure analysis is possible and highly useful, and students will be able to explore different approaches to quantifying microstructure using image analysis.

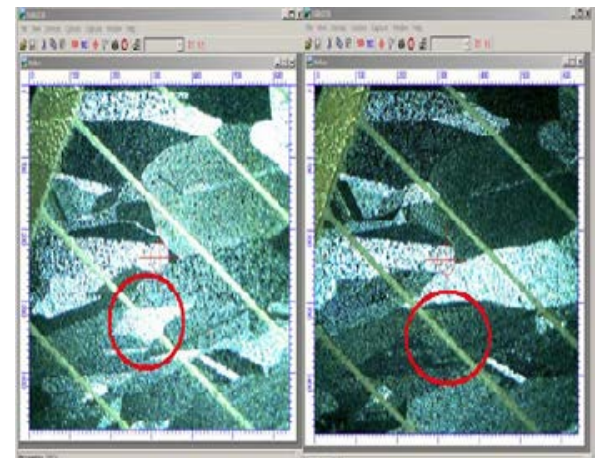

Figure 3: Solar cell illumination with white light from two different angles.

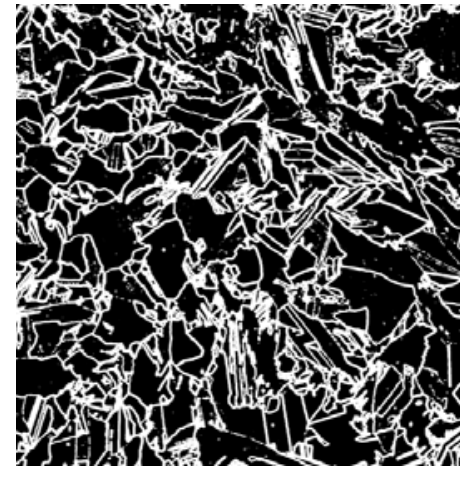

Figure 4: Skeletonized binary image showing grain boundaries. [FORSTER et al. 2009]

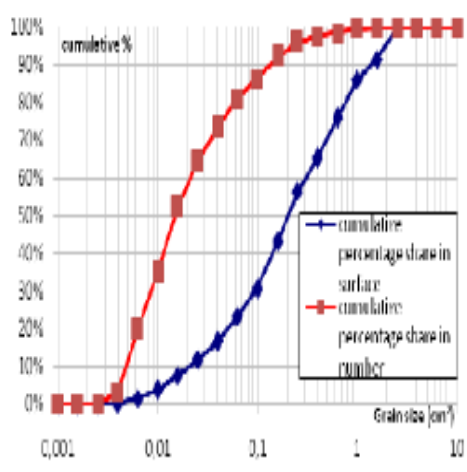

Figure 5: Cumulative distribution of grain sizes determined by image analysis. [FORSTER et al. 2009].

Figure 6 shows an image processing of solar cells on a conveyer belt, along with a laser light scattering device to measure surface roughness (Figure 7). One advantage of image capture is the potential high-throughput measurements and its non-contact nature. We emphasize this point to the students. That often image analysis is a faster alternative to other probing techniques, but that there is a trade-off in information and speed of measurement. An underlying feature of the modules is that measure-ments by different techniques, including image processing, need to be compared and validated. 


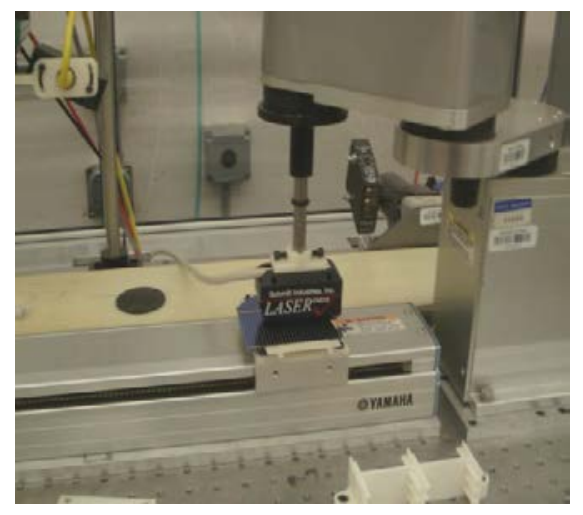

Figure 6: Conveyer-belt inspection of solar cells using CCD camera and laser light scattering.
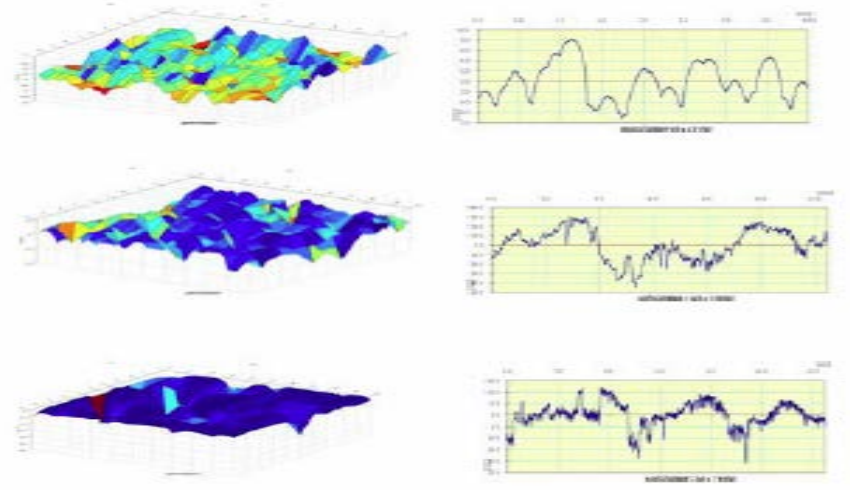

Figure 7: Comparing surface roughness topography by non-contact image processing and surface profiling with a stylus.

As a specific example, we measure surface roughness using scanning laser light scattering to map the solar cell, surface profiling with a contact stylus, white light interferometry imaging (Figures $\mathbf{8}$ and 9), atomic force microscopy, and image analysis with a CCD camera. Usually we can establish a 'gold standard' method for a particular feature under study, and then corroborate or compare measurements made by other imaging techniques. Students explore the limitations and comparative merits of each method. For instance, there are many algorithms to derive surface roughness from image data. A particularly simple method is based on the grey level histogram of images, from which statistics can be used to correlate images with the roughness determined by other techniques.

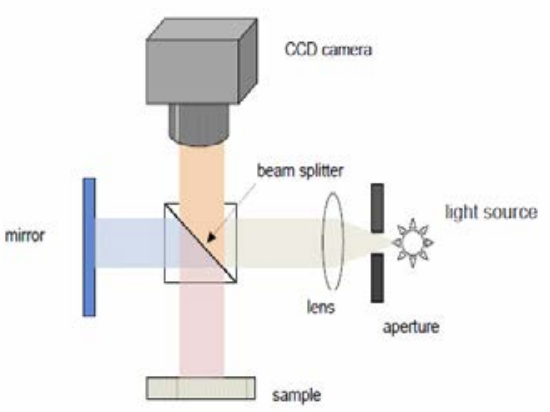

Figure 8: White light interfererometer for imaging surface roughness.

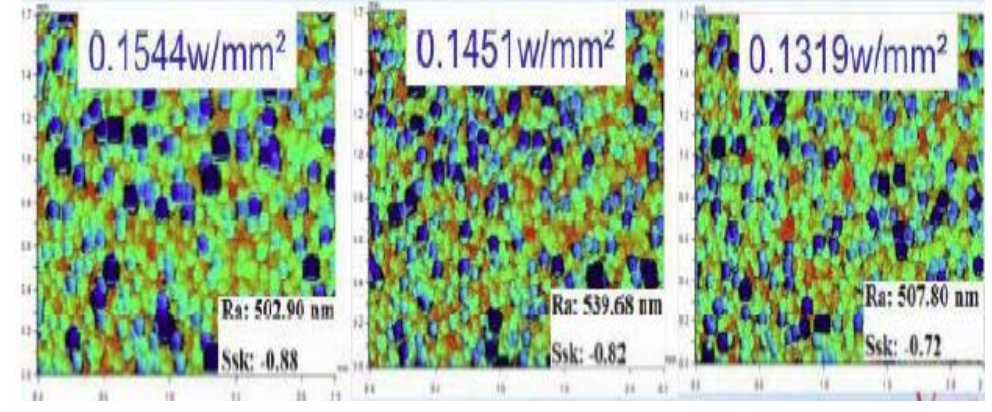

Figure 9: White light inteferometry measurements of silicon solar cells. From Veeco, Inc. product literature. 
As the solar cell is a luminous source (excited optically, electrically, or ultrasonically), it is amenable to many other imaging modalities: photoluminescence, electroluminescence, light-controlled transmission where infrared transmission through the solar cell is modulated by injected electrical charge due to free-carrier absorption effects. Although silicon is a so-called indirect bandgap semiconductor and is a poor luminescent material compared to other semiconductors (e.g., GaAs and GaN), it nevertheless exhibits quantum efficiencies of $\sim 0.1 \%$ which proves more than sufficient for room-temperarature, near-IR imaging. "Photons emitted by solar cells convey a wealth of information about materials properties, processing problems, and the quality and performance of the solar cell [KASEMANN et al. 2009]. There are established methods to determine voltage, surface potentials, Fermi levels, resistivity, thermoelectric effects, carrier trapping effects, and minority carrier recombination from luminescence solar cell images. Further, the emission spectrum, which can be determined by filtering the image, is characteristic of particular types of defects. This defect spectroscopy imaging is proving highly useful in diagnosing and improving semiconductor devices in general, and solar cells in particular.

Figure 10 shows a set-up for photoluminescence $(P L)$ imaging of solar cells. Figure 11 shows some examples of PL imaging of solar cells, which at the very least indicates some appreciable areal variation of luminescence intensity and underlying properties. Because PL is excited optically, with no electrical contacts (nor metallization, junction, doping) required, the $P L$ imaging can be done at all stages of fabrication-from silicon raw material, to wafers, to solar cells at various process steps, to finished modules.

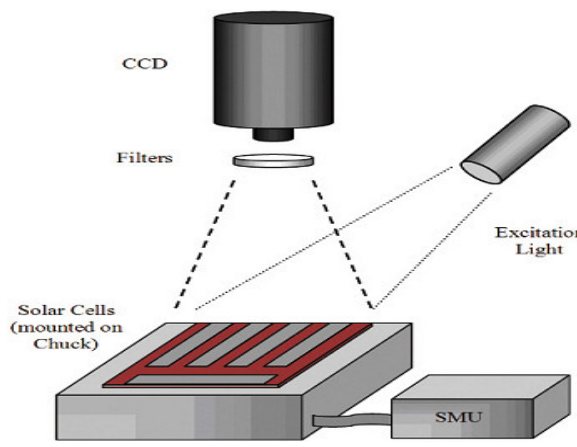

Figure 10: Solar cell photoluminescence imaging stage.
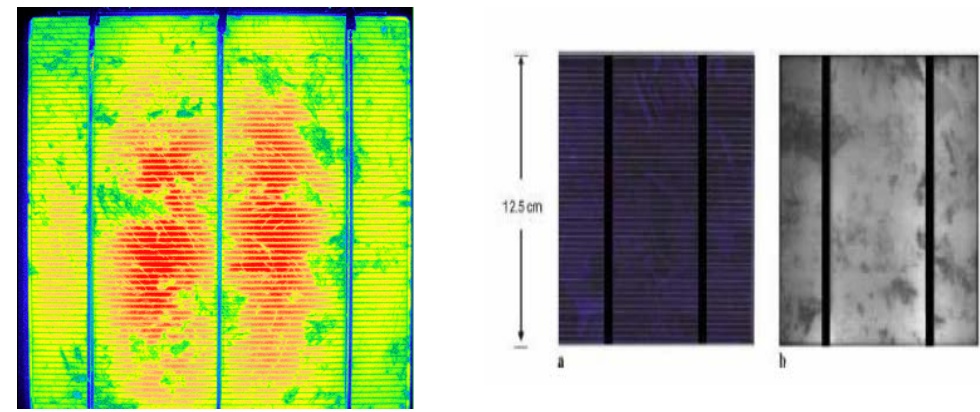

Figure 11: Photoluminescence images of multicrystalline silicon solar cells. From Fuyuki et al. [2009]. 
Lock-in thermography (Figure 12) is a relatively recent development in imaging and can be done for many imaging modalities. Lock-in techniques show the utility of quadrature (phase sensitive detection) and is the principle of modern MRI and NMR imaging, and are used increasingly in medical fields, and Non-Destructive thermography in the aerospace, electronics, and automobile industries. Temperature difference sensitivies in the 10 microKelvin range are feasible. Many thermal cameras have external triggering for lock-in imaging. Lock-in thermography allows the thermal waves (and reflections at interfaces and defects) to be imaged in pulse thermography methods, the analysis of which entails very interesting transient heat conduction analysis and image processing from the perspective of signal recovery and minimization of noise effects. Educational modules teaching lock-in thermography (as well as lock-in PL and EL optical imaging) of solar cells may be regarded as the capstone topics of the Solar Cell imaging program proposed here. Students will be working at the state-of-the-art. Finally, at a perhaps more practical and mundane application level, we show some thermal images of solar modules in the field (Figure 13), as a way of detecting hotspots, shunts, and aberrant solar cell performance. The program thus exposes students to imaging from raw materials inspection (multicrystalline silicon ingots) to the end-application of inspecting and maintaining solar modules mounted on roofs for example. Such imaging in the field plays an important role in sustainable infrastructure and energy efficiency.

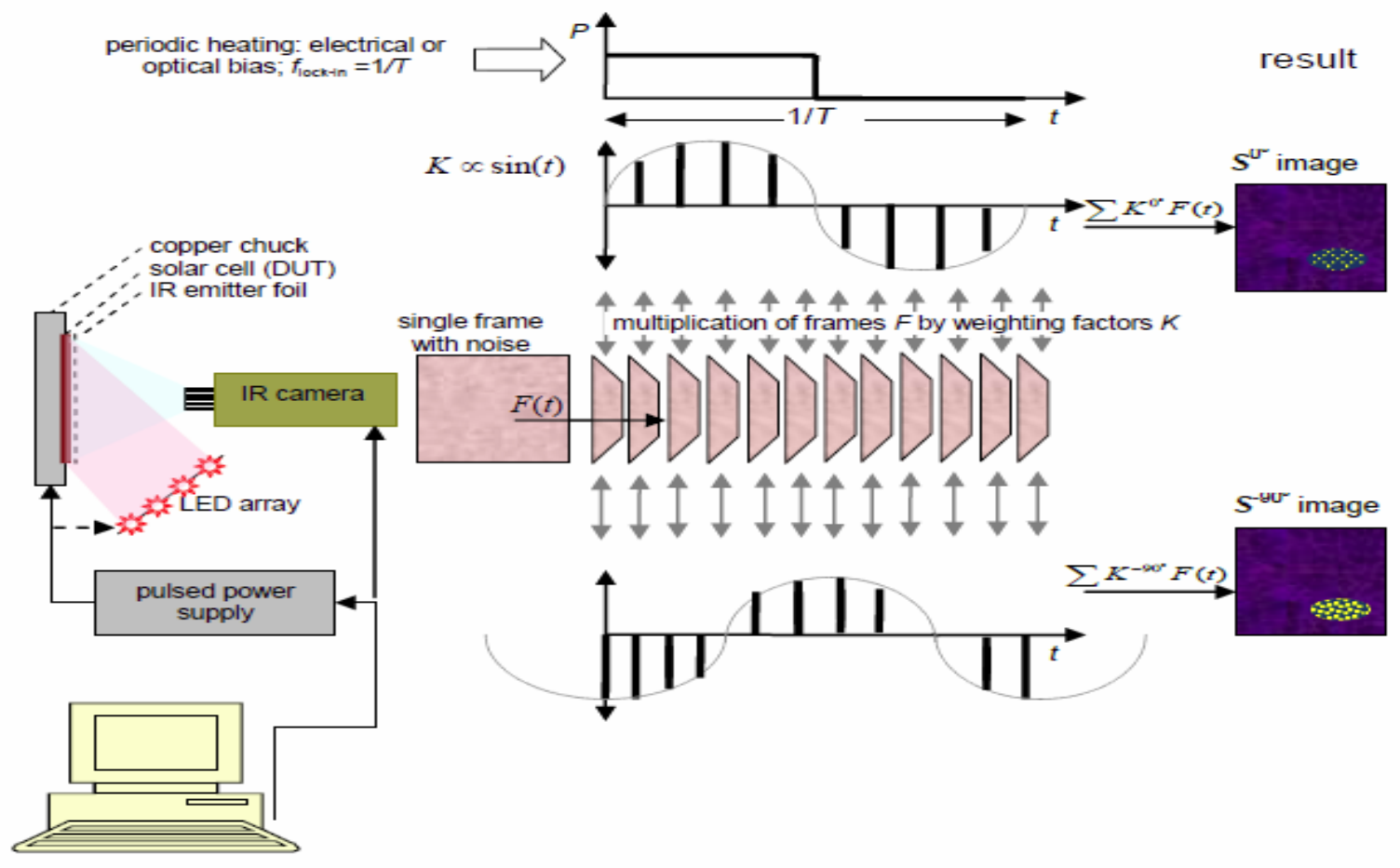

Figure 12: Lock-in thermography of solar cells. After BREITENSTEIN et al. [2012]. 

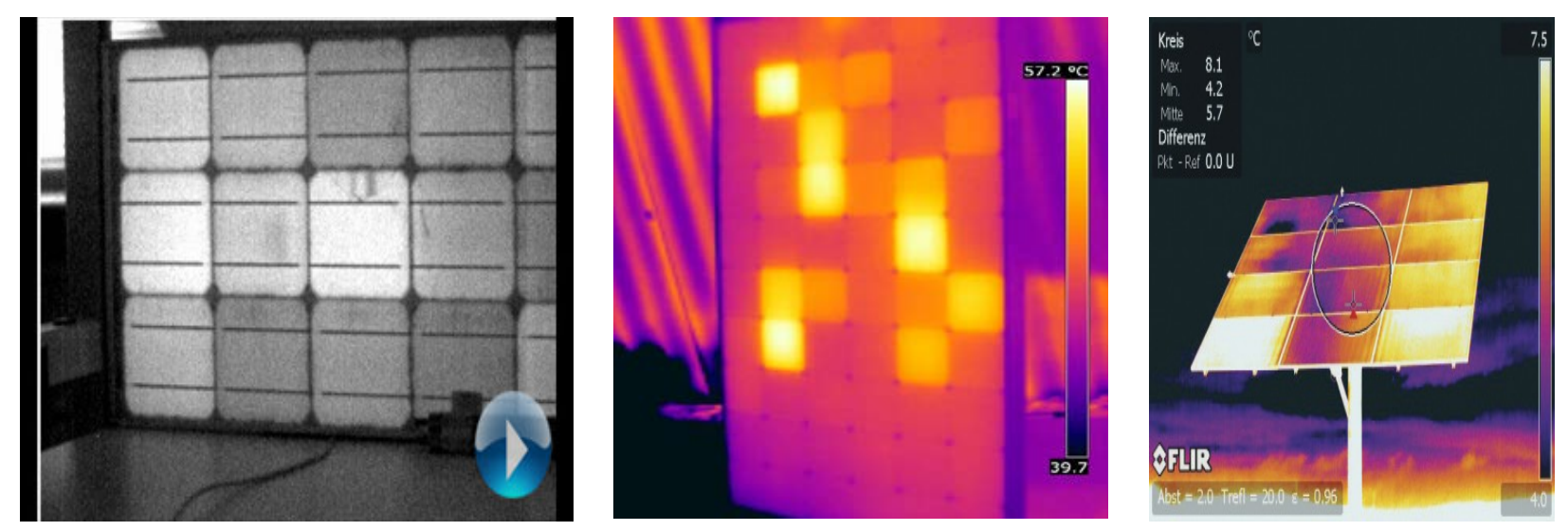

Figure 13: Thermal imaging of solar modules showing hot spots and anomalous solar cells. From Scanthermal, Inc. product literature.

\section{Educational Modules}

We will develop a series of educational modules that can be adopted by schools and universities to provide real-world instances of image capture and processing. Although each module is independent and self-contained, and be utilized singularly, instructors will be able to establish a coherent theme and progression from simple imaging case studies to more sophisticated, state-of-the-art techniques. Our plans for solar imaging modules include the following topical descriptions of each module:

1. Microstructure of Polycrystalline Silicon Wafers. Images of solar cells directly show the microstructure, particularly grain boundaries. Unlike metals, silicon reveals its grain structure without the need for any sample preparation. Students can apply many of algorithms (including ASTM protocols) used to parameterize microstructure from images. ASTM procedures using lineal and areal analysis, and similar methods, to determine grain size distributions and grain symmetry (e.g., equi-axed or elongated) will be implemented with MATLAB. The first modules provide the students with vision system particulars such as field of view, resolution, contrast, illumination, and the working distance and magnification of the camera [HOLLOWS and HOYER].

2. Basic Image Processing of Solar Cells. Images of solar cells are studied to detect surface uniformity and use standard image processing to measure solar cells features such as intact or broken grid lines, uniformity of AR coating, and microcracks in solar cells. We will also coat and dry cells with standardized solutions of particulates so that students can quantitatively assess defect distributions and areal yields.

3. White Light Interferometric Imaging of Solar Cells. White light interferometry produces images of solar cells that indicate surface texture [BLEWETT and NOVAK 
2010]. We have a VEECO white light interferometer that produces images similar to those shown in Figure 12, and can be used to quantify surface roughness. This can be compared with roughness measurements made by other methods.

4. Surface Roughness by Image Processing. Intensity histograms analysis can be correlated with roughness measured by a surface stylus profiler, laser light scattering, interferometry, or atomic force microscopy). There are numerous MATLAB algorithms in the trade and research literature on correlating parameters extracted from images with roughness measurements by nonimaging methods. CCD image based methods for assessing surface roughness have been described in detail by LuK et al. [1989], Rampira [2008],

5. Wetting Angles on Solar Cells. Drops of various liquids (water, electrolyte solutions, organics) on semiconductor surfaces show characteristic wetting angles that indicated oxidation, compound formation, and cleanliness [LOCKHANDE et al., 2003; ANGELO et al. 2007 and work cited therein]. Wetting angles can be measured accurately with a CCD camera and related to surface energies [SHETTY and RAMASAMY 2005].

6. Image Capture of Solar Cells on a Conveyer Belt with Robotic Sorting. An immensely important of image capture processing and analysis is to translate these techniques to the factory floor. Here the objective is to determining how discriminating and rapid image processing can be "on the fly"-see for example CHIOU et al. [2012],

7. Light-Beam Induced Current (LBIC) Imaging of Solar Cells. LBIC studies are the traditional means to map solar cell performance. Students in our lab used a robotic arm holding laser pointers (IR, red, green, and blue emission) to scan a solar cell and create a map of localized current generation and is sensitive to defects such as grain boundaries and shunts. Current variation is due to defective regions of the solar cell. This LBIC map can be compared with luminescence images of the cell.

8. Scanning a Solar Cell Surface with a Laser Light Scatter Surface Rough. The laser light scattering measurement is normally a point $(0.5 \mathrm{~mm})$ measurement. Our students used a programmed robotic arm to scan the solar cell and map surface roughness of solar cells-see also CHIOU et al. [2010] and LU and TIAN [2006], WALECKI et al. [2008], CHAUDHARY et al. [2005].

9. Assessment of Surface Cleanliness Using Fluorescent Imaging. Fluorescent dyes are absorbed on the surface of the solar cell and can be removed by rinsing. Fluorescent imaging, exciting with a UV light and detecting visible emission reveals the effectiveness of cleaning procedures [YAKOVLEV et al. 2005]. Students can perform a DOE (design of experiments) to optimize 
clean. Clean surfaces are crucial in many technologies including medical devices.

10. Advanced Image Processing for Solar Cells. More sophisticated solar cell imaging processing, especially for microcrack detection has been described by TSAl et al. [2010] who used anisotropic diffusion algorithms for crack inspection of textured silicon solar cells, and CHAO and TSAI [2010] for improved feature recognition, as well as imaging of laser speckle patterns [Wen 2012]

11. Hyperspectral Imaging for inspection is now enabled by the recent development of high-power, ultra-broadband "white light" laser-like sources (400 $\mathrm{nm}$ to 2 micron wavelengths, $6 \mathrm{~W}$ power) with coherence and collimation more like lasers than conventional light sources. These are now available from Fianium Inc (Eugene, OR and UK) for several thousand dollars with optical fiber outputs. Li et al. [2010] used these sources for hyperspectral imaging of defects in solar cells.

12. Photoluminescence (PL) Imaging of Solar Cells. The solar cell can be excited with a laser or flash lamp and (with filtering) the infrared photoluminescence can be imaged with a CCD camera with silicon or near-infrared InGaAs detector elements, or mid-IR MCT detector elements. The bibliography contains numerous references that describe PL imaging of solar cells.

13. Electroluminescence Imaging of Solar Cells. Similar to PL imaging, but luminescence is excited by electrical bias as shown in Figure 18, and described by FUYUKI et al. in detail.

14. Infrared Transmission Imaging of Solar Cells. Infrared transmission through the silicon solar cells can be imaged by placing a heated stage behind the solar cell, which acts as a blackbody source, see Figure 15 above. The free carriers create absorption effects such that the transmission thermal image maps the defects of the solar cell.

15. Measuring the Thermal Expansion of Solar Cell Laminates. EITNER et al [2011] measured thermal expansion of solar cell laminates using digital image correlation.

16. Thermal Imaging with Thermochromatic Films and Coatings. If a thermal camera is not available, a lower resolution, but nevertheless very iinformative thermography can be done by applying polymer-dispersed liquid crystal foils over the cell, which change color with temperature changes of $0.5^{\circ} \mathrm{C}$ or less. SCHMIDT and DIERKING [2001] and others have described imaging of solar cells using this method, which obviates the need for a thermal camera.

17. Lock-in Thermography of Solar Cells. The book by BREITENSTEIN and LEIGENKAMP, Lock-in Thermography for Funcitional Diagnostics of Electronic Components (Springer 2011) has detailed descriptions of solar cell thermography with lock-in thermal imaging. 
18. Lock-in themography with ultrasonic excitation. We have a state-of-the-art ultrasonic NDT lab with wavetanks for ultrasonic excitation of samples that can be thermally imaged, as per the methods of RANTALA et al. [1998] for watercoupled ultrasonic excitation.

Technical Requirements: The first series of educational modules utilize commercially available CMOS or CCD cameras, a set of samples with variegated characteristics, and a desktop workspace. Small lots of solar cells are available from many vendors (We identified at least 25 suppliers of small lots of solar cells with a Google search, e.g., PMaxx Scrap Educational Solar Cells, Edmond Scientific, Silicon Solar Innovative, etc...) A typical silicon solar cell is $12.5 \mathrm{~cm} \times 12.5 \mathrm{~cm}$ in area and costs on the order of $\$ 2$. It is instructive to image and analyze solar cells in various stages of fabrication. The proposers can provide schools with sample sets of a wide range of materials that have been collected over the last twenty years from research institutions and solar cell manufacturers. Alternatively, in cases where only finished solar cells are readily available, it is easy to "reverse engineer" the solar cell by selectively removing/etching the solar cell to remove the anti-reflection coating, surface passivation layers, metallization, and surface texture, and generate a retrospective series of samples representative of solar cells at different stages of the value chain. For instance, the AR coating and metallization can be stripped by abrading or immersing the solar cell in various acidic etches or solvents, recipes for which will be provided by us to module adopters. Also, solar cells can be intentionally damaged with scratches, chips, and such to explore imaging capabilities for detecting deviations and flaws. Moreover, many universities have active research programs exploring novel materials, processes, and device designs for solar cells and related technology, which can also serve as an inhouse source of samples. (In fact, it is our impression that many researchers are unaware or do not fully appreciate the full scope and capabilities of imaging as research tool, and may be eager to incorporate some of the techniques described here. This will provide an opportunity for engineering students using these educational modules to engage in basic science R\&D efforts.) More 'exotic' or 'cutting edge' solar cells and devices, such as the dye-sensitized $\mathrm{TiO}_{2}$ nanocrystalline solar cell, or organic, polymer, or quantum dot solar cells and LEDs are also good candidates for image processing We have imaged such novel devices with CCD cameras for quality control laboratory projects, showing for example the degradation of light-emission over the area of the device as a function of time.

Cameras: For optical imaging, CMOS and CCD webcams, digital microscopes, and machine vision cameras are suitable for most purposes, costing from $\$ 100$ to roughly $\$ 1000$. Many cameras have USB connections for easy computer interfacing. These silicon-based cameras have some responsivity in the near-IR and can be used in some cases for thermal imaging as well. Thermal imaging is most typically done with an IR camera in the 3-5 micron wavelength range (InGaAs detectors) or 8-12 micron 
wavelength range (MCT detectors). Prices for suitable thermal imaging cameras range from $\$ 1000$ to $\$ 2000$ at the low-end, to $\$ 10,000$ or more at the higher end, and include external triggering for lock-in modes of imaging. We mention that the prices continue to fall and capabilities (resolution, sensitivity, i.e., smallest resolved temperature differences, and image size) continue to improve, so that thermal imaging will be more pervasive in schools and industry.

Remote imaging online. For schools that cannot make expenditures for thermal cameras or other equipment, we will develop a complete remote access capability. The modules will utilize the capabilities already developed by us for remote access, webbased laboratories for quality control and robotics via machine vision (Figure 14) and CHIOU, MAUK, and DAVIS [2012] "Remote Solar Cell Surface Monitoring".
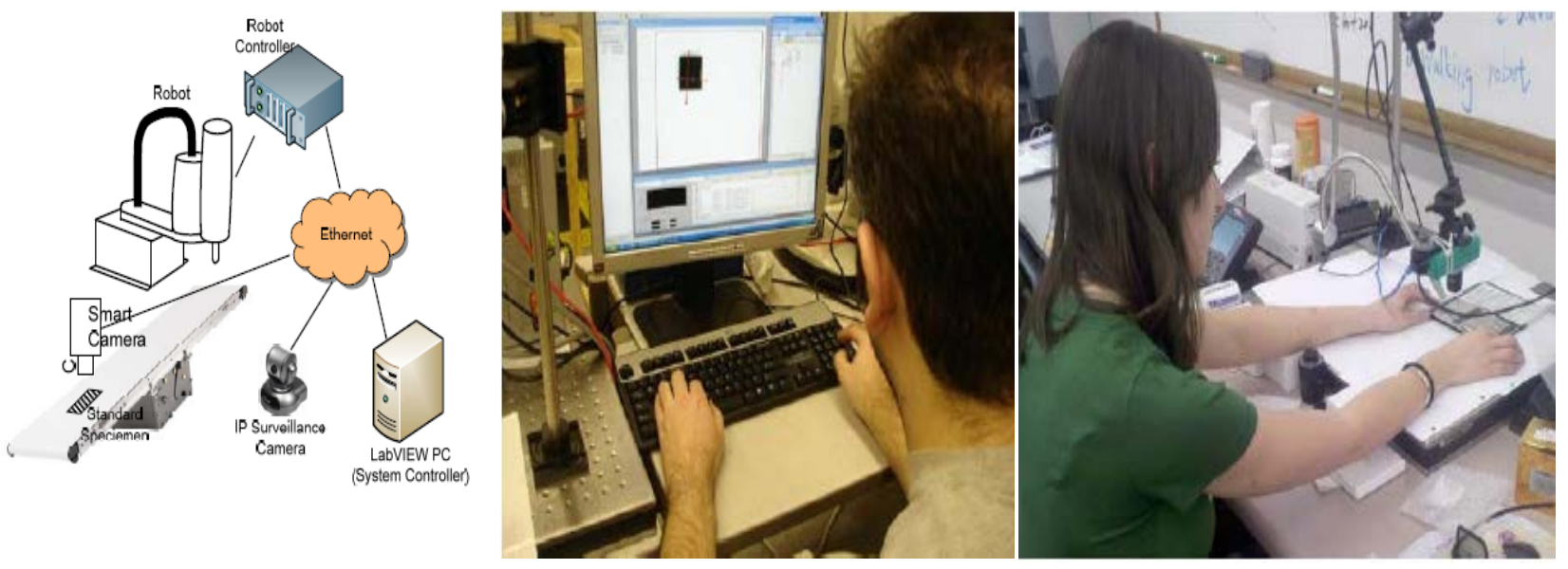

Figure 14: Remote imaging for web-based courses using IP addressable cameras. Students can remotely direct image capture sessions of solar cells.

Assessment and Evaluation. Evaluation of the project and data collection will begin soon after the start of the project. On the first meeting of each course, students will be administered a preliminary assessment test to gauge students' background and preparation for the course. For example, their knowledge of appropriate physics and chemistry will be evaluated. Based on the results of the test, students will be divided by groups according to the think-share-report-learn (TSRL) process, which will involve student peer coaching to help each other during the laboratory procedures.

The implementation of an integrated collection of formative and summative assessment strategies for the laboratory-based courses and projects in solar cell imaging will start in Year 1. Evaluation evidence will be generated through activities integrated into the natural flow of the project. Both qualitative and quantitative data will be collected from a variety of sources in a collective effort involving faculty, program participants, and other key stakeholders. To assess program performance, the evaluation first provides a detailed description of the program followed by a focus on three key areas: Program Implementation, Achievement of Program Outcomes, and Program Lessons Learned. 
The formative evaluation will provide evidence of the strengths and weaknesses of the project, informing the Pl's understanding of what works and what does not in order to assess project progress and success. Document analysis will interpret the quality and usefulness of materials produced in project delivery, while surveys will reveal attitudes and levels of understanding among participants-students and the ET/STEM faculty. Participant observations conducted in the form of self-assessment journals and logs, as well as more objective professional non-participant observations, will reveal underlying project and personal dynamics. Knowledge outcomes and skill development in the form of instructional materials and strategies, as well as applied knowledge products will be evidenced in the form of content generated in the course. Data and statistical analysis generated by students in the solar cell imaging exercises and projects will be used by instructors in assessing the progress of the students in learning the course content. Thus, through each phase of the course, a statistical database is developed and augmented about processes and products. The preliminary and intermediate analyses made by the students also provide numerous metrics to the instructors, which can be used to monitor the competency and understanding of the students' use and application of Six Sigma techniques. For example, student data collection results can be evaluated based on statistical tests for normality, repeatability and reproducibility, correlation with process variables, and analysis of variance (ANOVA). A summative evaluation will assess the quality and impact of an implemented project based on the students' final presentations, including corrections of the collected results and conclusions. Proposed summative evaluation questions include:

- To what extent did participants use what they were taught in their own activities?

- Which topics and techniques were most often (or least often) incorporated?

- To what extent did participants share their recently acquired knowledge and skills

- To what extent was there an impact on participating students? Have STEM faculty become more (or less) positive about integrating experiences and making such experiences an important component of their student's future career?

- Did changes occur in the overall perception and behavior of participants?

- What were the obstacles, if any, to the introduction of changes?

- Is the overall program consistent with stated goals and objectives?

Standard course evaluation forms will ask students to compare their level of competence in areas identified in the course objectives at the end of the course to their level before taking the course. This provides immediate feedback on the success of the course in meeting its objectives. The form will include the Likert-type questions, which assess the students' perception of their confidence, knowledge, and competence.

\section{Acknowledgements}

This work was supported by "Solar Cell Imaging: A Gateway for Visualization and Learning in STEM Disciplines" NSF TUES Award DUE-1245872 (June 2013 to May 2015). 